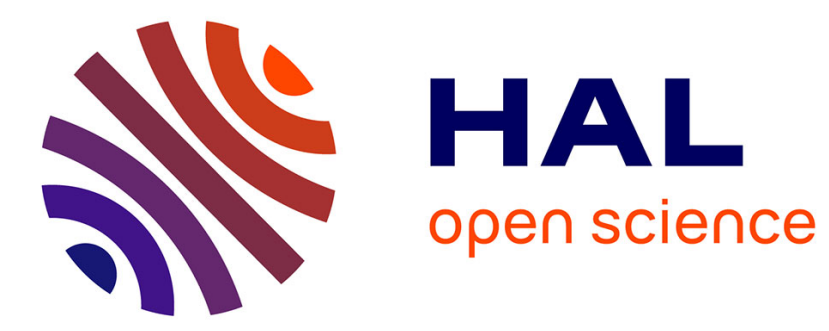

\title{
Loss of SMARCB1 expression in colon carcinoma
}

S. Melloul, J-F Mosnier, J. Masliah-Planchon, C. Lepage, K. Le Malicot, J-M Gornet, J. Edeline, D. Dansette, P. Texereau, O. Delattre, et al.

\section{To cite this version:}

S. Melloul, J-F Mosnier, J. Masliah-Planchon, C. Lepage, K. Le Malicot, et al.. Loss of SMARCB1 expression in colon carcinoma. Cancer Biomarkers, 2020, 27 (3), pp.399-406. 10.3233/CBM-190287 . hal-02798007

\section{HAL Id: hal-02798007 https://hal-univ-rennes1.archives-ouvertes.fr/hal-02798007}

Submitted on 12 Jun 2020

HAL is a multi-disciplinary open access archive for the deposit and dissemination of scientific research documents, whether they are published or not. The documents may come from teaching and research institutions in France or abroad, or from public or private research centers.
L'archive ouverte pluridisciplinaire HAL, est destinée au dépôt et à la diffusion de documents scientifiques de niveau recherche, publiés ou non, émanant des établissements d'enseignement et de recherche français ou étrangers, des laboratoires publics ou privés. 


\title{
Loss of SMARCB1 expression in colon carcinoma
}

\author{
S. Melloul ${ }^{\mathrm{a}}$, J.-F. Mosnier ${ }^{\mathrm{b}}$, J. Masliah-Planchon ${ }^{\mathrm{c}, \mathrm{d}, \mathrm{e}}$, C. Lepage $^{\mathrm{f}, \mathrm{g}}$, K. Le Malicot $^{\mathrm{g}, \mathrm{h}}$, J.-M. Gornet ${ }^{\mathrm{i}}$, \\ J. Edeline ${ }^{\mathrm{j}}$, D. Dansette ${ }^{\mathrm{b}}$, P. Texereau ${ }^{\mathrm{k}}$, O. Delattre ${ }^{\mathrm{c}, \mathrm{d}, \mathrm{e}}$, P. Laurent Puig ${ }^{\mathrm{l}, \mathrm{m}}, \mathrm{J}$. Taieb ${ }^{\mathrm{l}, \mathrm{m}}$ and \\ J.-F. Emile ${ }^{\mathrm{a}, \mathrm{b}, \mathrm{c}, \mathrm{d}, \mathrm{e}, \mathrm{f}, \mathrm{g}, \mathrm{h}, \mathrm{i}, \mathrm{j}, \mathrm{k}, \mathrm{l}, \mathrm{m}, \mathrm{n}, *}$ \\ ${ }^{a}$ Department of Pathology, Ambroise Paré Hospital, APHP, Boulogne, France \\ ${ }^{\mathrm{b}}$ Department of Pathology, Hotel Dieu, Nantes, France \\ 'Somatic Genetic Unit, Institut Curie, Paris, France \\ ${ }^{d}$ Paris-Sciences-Lettres, Institut Curie Research Center, INSERMU830, Paris, France \\ SIREDO, Institut Curie, Paris, France \\ ${ }^{\mathrm{f}}$ François Mitterrand University Hospital, Dijon, France \\ ${ }^{g}$ EPICAD INSERM LNC-UMR 1231, University of Burgundy and Franche-Comté, Besançon, France \\ ${ }^{\mathrm{h}}$ Francophone Society of Digestive Cancer, Dijon, France \\ ${ }^{i}$ St Louis Hospital, APHP, Paris, France \\ ¡Eugène Marquis Center, Rennes, France \\ ${ }^{\mathrm{k}}$ Layne Hospital Center, Mont-de-Marsan, France \\ ${ }^{1}$ G. Pompidou European Hospital, APHP, Paris, France \\ m INSERM UMR-S1147, Paris, France \\ ${ }^{\mathrm{n}}$ EA4340-BCOH, Versailles SQY University, Paris-Saclay University, Boulogne, France
}

\begin{abstract}
SMARCB1 is a tumor suppressor gene, which is part of SWI/SNF complex involved in transcriptional regulation. Recently, loss of SMARCB1 expression has been reported in gastrointestinal carcinomas. Our purpose was to evaluate the incidence and prognostic value of SMARCB1 loss in colon carcinoma (CC).

Patients with stage III CC $(n=1695)$, and a second cohort of 23 patients with poorly differentiated CC were analyzed. Immunohistochemistry for SMARCB1 was performed on tissue microarrays, and cases with loss of expression were controlled on whole sections. Loss of SMARCB1 was compared with the clinico-pathological and molecular characteristics, and the prognostic value was evaluated.

Loss of SMARCB1 was identified in 12 of $1695(0.7 \%)$ patients with stage III CC. Whole section controls showed a complete loss in only one of these cases, corresponding to a medullary carcinoma. SMARCB1 loss was not associated with histological grade, tumor size nor survival. In the cohort of poorly differentiated CC, we detected 2/23 (8.7\%) cases with loss of SMARCB1; one was rhabdoid while the other had medullary and mucinous histology. These 2 cases were deficient for MisMatched Repair (dMMR) and mutated for $B R A F$.

SMARCB1 loss is rare in stage III CC, but appears more frequent in poorly differentiated CC.

Keywords: SMARCB1, colon carcinoma, BRAF V600E, mismatch repair deficiency

\section{Abbreviations}

CC colon carcinoma

CRC colorectal carcinoma

dMMR deficient for MisMatched Repair

IHC Immunohistochemistry

FFPE frozen tissue and formalin-fixed paraffin-embedded tissue

MSI Microsatellite instability

PETACC8 The Pan-European Trials in Alimentary traCt Cancer

${ }^{*}$ Corresponding author: J.-F. Emile, Service de Pathologie, Hôpital Ambroise Paré, 9 Av Ch. De Gaulle, 92104 Boulogne, France. E-mail: jean-francois.emile@uvsq.fr.

\section{PFS}




\begin{tabular}{|ll}
\hline SMARCB1 & $\begin{array}{l}\text { SWI/SNF-related matrix-associated } \\
\text { actin-dependent regulator of } \\
\text { chromatin subfamily B member 1 } \\
\text { Tissue microarray }\end{array}$ \\
&
\end{tabular}

\section{Background}

SMARCB1 (SWI/SNF-related matrix-associated actin-dependent regulator of chromatin subfamily $B$ member 1) is a chromatin-remodeling gene and a tumor suppressor gene located on chromosome 22q11 [6, 8,18]. SMARCB1 encodes for the protein SMARCB1 which is part of the multimolecular SWI/SNF complex involved in transcriptional regulation. The biallelic inactivation of $S M A R C B 1$ was first described in pediatric rhabdoid tumors, and is the main oncogenic mechanism. Then, loss of SMARCB1 protein expression has been reported in a range of malignant neoplasms including pediatric rhabdoid tumors (atypical teratoid/rhabdoid tumors of the nervous system and malignant rhabdoid tumours) [5], epithelioid sarcomas [8,9] and renal medullary carcinoma [7]. SMARCB1 mutation/inactivation has been reported to be correlated with the loss of nuclear expression of SMARCB1 determined by immunohistochemistry (IHC) [8], and IHC for SMARCB1 is already used by pathologists to confirm the diagnosis of rhabdoid tumor.

Recently, SMARCB1 loss has been described in series of gastrointestinal adenocarcinomas with rhabdoid morphology, including colon adenocarcinomas (CC) $[1,11,12,19]$. Wang showed that the loss of SMARCB1 in CC was rare $(0.46 \%)$, and associated with higher histological grade, larger tumor size, lower survival, MSI and BRAF V600E status $(p<$ 0.001). However, the frequency and prognostic value of SMARCB1 loss according to the stages of CC remains unclear. We therefore wanted to evaluate the incidence as well as the clinical pathological and molecular associations of SMARCB1 loss in a large, homogenous and well characterized cohort of patients with stage III CC, included in a prospective clinical trial and all receiving FOLFOX adjuvant.

\section{Methods}

\subsection{Patients}

The stage III CC series corresponds to 2043 patients included in the PETACC8 study [13], who signed a specific consent for translational research and for whom FFPE samples were available. PETACC8 is an open, randomized, controlled, multi-center, multinational, phase 3 study in 18-75 years old patients, who underwent complete resection of stage III colon carcinoma (clinical trials \# NCT00265811). Patients were randomized to receive adjuvant therapy: 6 months of FOLFOX 4 or FOLFOX and Cetuximab. The study was done in accordance with the Declaration of Helsinki (amended 2000) and the International Conference on Harmonization of Technical Requirements of Pharmaceuticals for Human Use (ICH) Note for Guidance on Good Clinical Practice and approved by the appropriate Ethics Committees.

The second series consists in 23 patients with poorly differentiated CC treated in Ambroise Paré hospital (Boulogne, France) or Laennec hospital (Nantes, France). In particular, the serie of Nantes consisted in 17 medullary carcinomas.

\subsection{SMARCB1 immunohistochemistry}

Immunohistochemistry for SMARCB1 was performed using a mouse monoclonal antibody (clone 25/BAF47, BD Bioscience). SMARCB1 staining was interpreted by two observers (JFE, SM) who were blinded to clinical, pathological and molecular data at the time of analysis. The loss of SMARCB1 expression was established when the nuclei of tumor cells were not stained and the adjacent stromal cells, inflammatory and/or endothelial component as well as normal colonic cells (positive control) were positive. Normal liver tissue was used as a positive control for each TMA. We used an evaluation score of the loss of SMARCB1 in 3 categories according to the percent of positive tumor cells [19], and 2 subcategories: focal or diffuse loss. We only considered the percentage of labeled cells with SMARCB1 antibody, and not the intensity of the marking (Fig. 1 and Supplementary file 1). The score was noted "not interpretable" when neoplastic cells and also internal controls were negative for SMARCB1.

For the first series of patients, IHC of all cases was performed on tissue micro array (TMA) sections, and those who had focal or diffuse total loss of SMARCB1 underwent staining on whole sections. Immunohistochemistry on whole sections was interpreted as diffusely negative when there was negative staining in all neoplastic cells and a positive control in non-neoplastic cells (Fig. 2).

We then analyzed the expression of SMARCB1 in whole sections in the small independent cohort of 


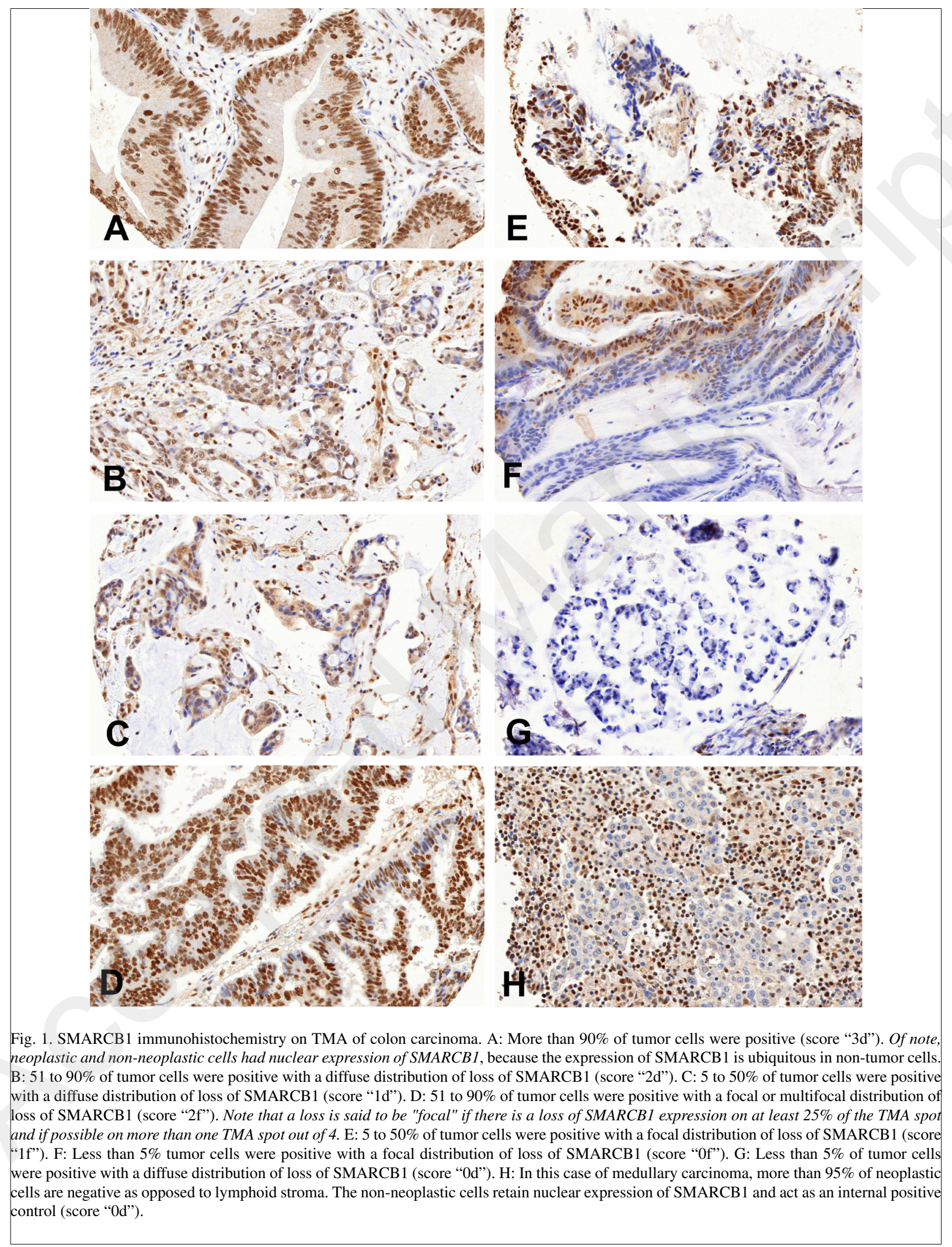




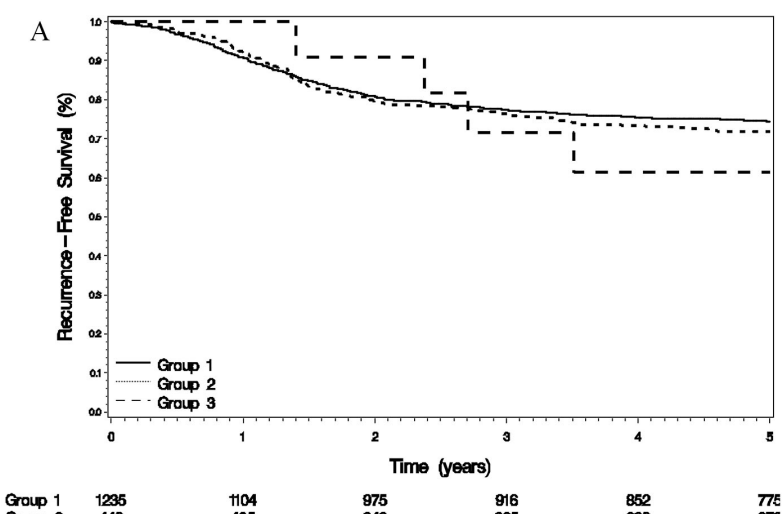

B

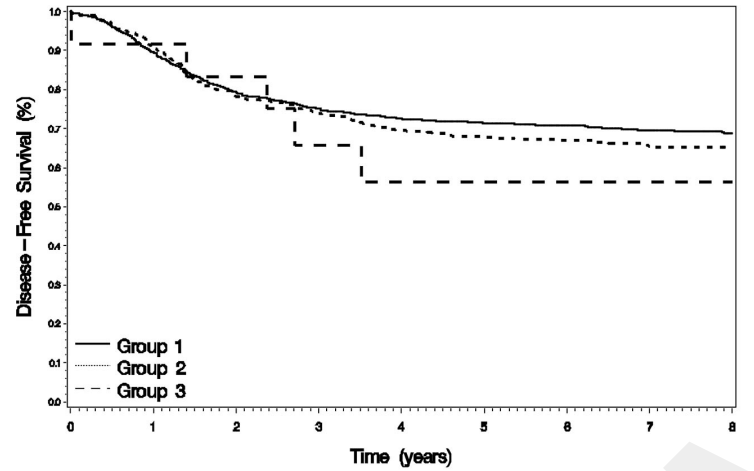

C
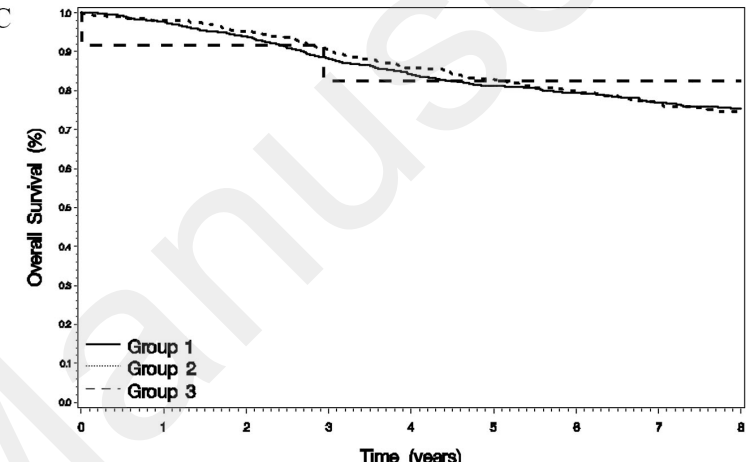

42

Fig. 2. Kaplan-Meir survival curves comparing SMARCB1 negative (group 3) and SMARCB1 positive CC (groups 1 and 2). A: Recurrence-Free survival according to SMARCB1 status. B: DFS according to SMARCB1 status. C: Overall survival according to SMARCB1 status.

23 patients treated in Boulogne or Nantes for poorly differentiated CC.

\subsection{Statistic analyses}

Prognostic and molecular status (mismatch repair (MMR) and BRAF V600E mutation) and detailed methods used to determine this status have been previously reported in the literature $[4,14,15]$. The association between CC clinicopathological and molecular variables and SMARCB1 expression were individually examined and binary logistic regression modeling was used. Kaplan Meier analysis and Cox regression modeling were employed to examine the impact of SMARCB1 expression with overall survival. A $p<$ 0.05 was taken as significant. For statistical analyzes we used 3 groups as follows:

Group 1: 3d (conserved expression of SMARCB1 on more than $90 \%$ of tumor cells)

Group 2: 1 and 2 (labeling of SMARCB1 on 5 to $90 \%$ of tumor cells)

Group 3: 0 (total loss of SMARCB1)

\subsection{SMARCB1 molecular analysis}

Tumor DNA was extracted from FFPE samples after histology control and selection of areas containing more than $80 \%$ of tumor cells. The whole coding sequence as well as the previously described hotspot in the first intron of SMARCBI were analyzed using next generation sequencing (NGS). Libraries obtained with Agilent SureSelect XT-HS preparation kit were sequenced on a NovaSeq 6000 system. The bioinformatics analysis included a variant calling using Varscan2 (v2.4.3) and TransIndel for intermediate insertion/deletion and a copy number profile using Facets (v0.5.1) with a sex-match control as reference $[10,16]$

\section{Results}

SMARCB1 immunostaining on TMA was interpretable in 1695 out of $2043(82.9 \%)$ patients with stage III CC of the PETACC 8 trial. In the 1695 (18.6\%) analyzed cases, 315 were poorly or undifferentiated 
Table 1

Correlation of loss of SMARCB1 to clinicopathological and molecular data. Clinical and pathological characteristics of patients with loss of SMARCB1 (group 3) compared with those of patients without loss of SMARCB1 (groups 1 or 2)

\begin{tabular}{|c|c|c|c|c|c|c|}
\hline & & $\begin{array}{c}\text { Group 1 } \\
(N=1235)\end{array}$ & $\begin{array}{l}\text { Group 2 } \\
(N=448)\end{array}$ & $\begin{array}{l}\text { Group } 3 \\
(N=12)\end{array}$ & $\begin{array}{c}\text { Total } \\
(N=1695)\end{array}$ & $p$-value \\
\hline \multirow[t]{3}{*}{ Gender } & $n$ & 1235 & 448 & 12 & 1695 & $X^{2}: 0.0759$ \\
\hline & Male & $688(55.7 \%)$ & $274(61.2 \%)$ & $5(41.7 \%)$ & $967(57.1 \%)$ & \\
\hline & Female & $547(44.3 \%)$ & $174(38.8 \%)$ & $7(58.3 \%)$ & $728(42.9 \%)$ & \\
\hline \multirow{3}{*}{ Age (Class) } & $n$ & 1235 & 448 & 12 & 1695 & $X^{2}: 0.5896$ \\
\hline & $\leqslant 70$ ans & $1108(89.7 \%)$ & $407(90.8 \%)$ & $10(83.3 \%)$ & $1525(90.0 \%)$ & \\
\hline & $>70$ ans & $127(10.3 \%)$ & $41(9.2 \%)$ & $2(16.7 \%)$ & $170(10.0 \%)$ & \\
\hline \multirow[t]{5}{*}{ Age } & $n$ & 1235 & 448 & 12 & 1695 & KW: $\mathbf{0 . 1 5 6 5}$ \\
\hline & Moy (SD) & $59.06(9.54)$ & $59.91(9.23)$ & $54.92(14.08)$ & $59.26(9.50)$ & \\
\hline & Médiane & 60.00 & 62.00 & 59.50 & 60.00 & \\
\hline & Q1; Q3 & $53.00 ; 67.00$ & $54.00 ; 67.00$ & $46.00 ; 64.50$ & $54.00 ; 67.00$ & \\
\hline & Min; Max & $23.00 ; 75.00$ & $19.00 ; 75.00$ & $28.00 ; 72.00$ & $19.00 ; 75.00$ & \\
\hline WHO performance & $n$ & 1190 & 436 & 11 & 1637 & $X^{2}: \mathbf{0 . 8 1 6 0}$ \\
\hline \multirow[t]{4}{*}{ status } & 0 & $966(81.2 \%)$ & $354(81.2 \%)$ & $10(90.9 \%)$ & $1330(81.2 \%)$ & \\
\hline & 1 & $218(18.3 \%)$ & $82(18.8 \%)$ & $1(9.1 \%)$ & $301(18.4 \%)$ & \\
\hline & 2 & $5(0.4 \%)$ & $0(0.0)$ & $0(0.0)$ & $5(0.3 \%)$ & \\
\hline & 3 & $1(0.1 \%)$ & $0(0.0)$ & $0(0.0)$ & $1(0.1 \%)$ & \\
\hline \multirow[t]{4}{*}{ Localisation } & $n$ & 1231 & 444 & 12 & 1687 & $X^{2}: 0.0624$ \\
\hline & Left localization & $711(57.8 \%)$ & $291(65.5 \%)$ & $7(58.3 \%)$ & $1009(59.8 \%)$ & \\
\hline & Right localization & $506(41.1 \%)$ & $147(33.1 \%)$ & $5(41.7 \%)$ & $658(39.0 \%)$ & \\
\hline & Both sides & $14(1.1 \%)$ & $6(1.4 \%)$ & $0(0.0)$ & $20(1.2 \%)$ & \\
\hline Bowel obstruction & $n$ & 1235 & 448 & 12 & 1695 & $X^{2}: \mathbf{0 . 5 5 0 7}$ \\
\hline \multirow[t]{2}{*}{ and perforation } & Bowel obstruction and/or perforation & $234(18.9 \%)$ & $90(20.1 \%)$ & $1(8.3 \%)$ & $325(19.2 \%)$ & \\
\hline & No bowel obstruction and no perforation & $1001(81.1 \%)$ & $358(79.9 \%)$ & $11(91.7 \%)$ & $1370(80.8 \%)$ & \\
\hline Histopathology & $n$ & 1234 & 448 & 12 & 1694 & $X^{2}: 0.7747$ \\
\hline \multirow[t]{4}{*}{ grading } & Well differentiated & $246(19.9 \%)$ & $102(22.8 \%)$ & $0(0.0)$ & $348(20.5 \%)$ & \\
\hline & Moderately differentiated & $743(60.2 \%)$ & $261(58.3 \%)$ & $8(66.7 \%)$ & $1012(59.7 \%)$ & \\
\hline & Poorly differentiated & $227(18.4 \%)$ & $77(17.2 \%)$ & $4(33.3 \%)$ & $308(18.2 \%)$ & \\
\hline & Undifferentiated & $5(0.4 \%)$ & $2(0.4 \%)$ & $0(0.0)$ & $7(0.4 \%)$ & \\
\hline \multirow[t]{3}{*}{ PN classification } & $n$ & 1235 & 448 & 12 & 1695 & $X^{2}: 0.0143$ \\
\hline & $\mathrm{pN} 1$ & $783(63.4 \%)$ & $260(58.0 \%)$ & $11(91.7 \%)$ & $1054(62.2 \%)$ & \\
\hline & $\mathrm{pN} 2$ & $452(36.6 \%)$ & $188(42.0 \%)$ & $1(8.3 \%)$ & $641(37.8 \%)$ & \\
\hline \multirow{7}{*}{ PT classification } & $n$ & 1235 & 448 & 12 & 1695 & $X^{2}: 0.6254$ \\
\hline & pT1 & $30(2.4 \%)$ & $13(2.9 \%)$ & $0(0.0)$ & $43(2.5 \%)$ & \\
\hline & pT2 & $81(6.6 \%)$ & $30(6.7 \%)$ & $2(16.7 \%)$ & $113(6.7 \%)$ & \\
\hline & pT3 & $870(70.4 \%)$ & $301(67.2 \%)$ & $9(75.0 \%)$ & $1180(69.6 \%)$ & \\
\hline & pT4 & $253(20.5 \%)$ & $103(23.0 \%)$ & $1(8.3 \%)$ & $357(21.1 \%)$ & \\
\hline & pTis & $0(0.0)$ & $1(0.2 \%)$ & $0(0.0)$ & $1(0.1 \%)$ & \\
\hline & pTx & $1(0.1 \%)$ & $0(0.0)$ & $0(0.0)$ & $1(0.1 \%)$ & \\
\hline \multirow[t]{4}{*}{ VELI } & $n$ & 1235 & 448 & 12 & 1695 & $X^{2}: 0.2864$ \\
\hline & Vascular invasion or lymphatic infiltration & $685(55.5 \%)$ & $265(59.2 \%)$ & $6(50.0 \%)$ & $956(56.4 \%)$ & \\
\hline & $\begin{array}{l}\text { No vascular invasion and no lymphatic } \\
\text { infiltration }\end{array}$ & $350(28.3 \%)$ & $128(28.6 \%)$ & $3(25.0 \%)$ & $481(28.4 \%)$ & \\
\hline & Missing & $200(16.2 \%)$ & $55(12.3 \%)$ & $3(25.0 \%)$ & $258(15.2 \%)$ & \\
\hline Combined & $n$ & 1151 & 407 & 9 & 1567 & $X^{2}: 0.1416$ \\
\hline \multirow[t]{3}{*}{ RAS/BRAF } & Double WT & $448(38.9 \%)$ & $180(44.2 \%)$ & $2(22.2 \%)$ & $630(40.2 \%)$ & \\
\hline & RAS mutant & $569(49.4 \%)$ & $183(45.0 \%)$ & $7(77.8 \%)$ & $759(48.4 \%)$ & \\
\hline & BRAF mutant & $134(11.6 \%)$ & $44(10.8 \%)$ & $0(0.0)$ & $178(11.4 \%)$ & \\
\hline \multirow[t]{3}{*}{ RAS } & $n$ & 1148 & 405 & 9 & 1562 & $\mathrm{X}^{2}: 0.0651$ \\
\hline & Wild-Type & $578(50.3 \%)$ & $222(54.8 \%)$ & $2(22.2 \%)$ & $802(51.3 \%)$ & \\
\hline & Mutated & $570(49.7 \%)$ & $183(45.2 \%)$ & $7(77.8 \%)$ & $760(48.7 \%)$ & \\
\hline \multirow[t]{4}{*}{ BRAF } & $n$ & 1182 & 426 & 10 & 1618 & $X^{2}: 0.0006$ \\
\hline & Wild-Type & $1045(88.4 \%)$ & $378(88.7 \%)$ & $8(80.0 \%)$ & $1431(88.4 \%)$ & \\
\hline & Mutated & $117(9.9 \%)$ & $41(9.6 \%)$ & $0(0.0)$ & $158(9.8 \%)$ & \\
\hline & Test failure & $20(1.7 \%)$ & $7(1.6 \%)$ & $2(20.0 \%)$ & $29(1.8 \%)$ & \\
\hline \multirow[t]{3}{*}{ MMR status } & $n$ & 1235 & 448 & & 1695 & $X^{2}: 0.0698$ \\
\hline & pMMR & $1103(89.3 \%)$ & $416(92.9 \%)$ & $10(83.3 \%)$ & $1529(90.2 \%)$ & \\
\hline & dMMR & $132(10.7 \%)$ & $32(7.1 \%)$ & $2(16.7 \%)$ & $166(9.8 \%)$ & \\
\hline
\end{tabular}


CC. There was no significant difference in outcome according of histological grade (Table 1).

Tumors from 12 of these patients (group $3=0.7 \%$ ) were had either focal $(n=9)$ or diffuse $(n=3)$ total loss of SMARCB1 on TMA and underwent IHC on whole sections. These tumors were all moderately $(8 / 12)$ or poorly $(3 / 12)$ differentiated. Whole section controls disclosed a complete loss in only one case, corresponding to a medullary carcinoma.

The clinicopathological and molecular features of the CC with and without SMARCB1 loss are presented in Table 1. Diffuse or focal SMARCB1 loss was not associated with clinical characteristics, histological grade, tumor size, mismatch repair deficiency nor $B R A F$ mutation.

Loss of SMARCB1 was not associated with poorer survival (Supplementary file 2, Fig. 2).

Surprisingly, group 1 (with a conservation of SMARCB1 expression) had a worse node status. But this is irrelevant clinically and is probably due to a bias related to the low number of cases in group 3.

Among the 23 poorly or undifferentiated $\mathrm{CC}$ of the second cohort, we detected $2(8.7 \%)$ cases with total focal loss of SMARCB1; one was rhabdoid and the other poorly differentiated (medullary and mucinous). These 2 cases were deficient for MMR and mutated for $B R A F$ (Supplementary files 3 and 4).

Molecular mechanisms responsible for the loss of SMARCB1 expression were investigated in the two cases with total and focal loss. NGS allowed a mean coverage of $699 \mathrm{X}$ and $488 \mathrm{X}$ over the whole design in those two cases and a minimal coverage of $100 \mathrm{X}$ for SMARCB1, but did not reveal any pathogenic variant in the coding sequence, nor at the hotspot within intron 1 of SMARCB1. Copy number profile also assessed by NGS revealed neither large deletion nor duplication in SMARCB1. Interestingly, we detected a large region of copy-neutral loss of heterozygoty ( $\mathrm{LOH}$ ) encompassing the whole SMARCBI locus in one of the cases.

\section{Discussion}

We detected a loss of SMARCB1 in 12 out 1695 $(0.7 \%)$ cases with stage III CC included in the prospective international clinical trial PETACC8 and did not found any correlation with clinical, histologic or molecular characteristics, nor with survival. The frequency of loss of SMARCB1 was higher in our second cohort of 23 patients with poorly differentiated $\mathrm{CC}$.

The incidence of loss of SMARCB1 in CC was initially reported to be $11 \%(15 / 134)$ [11]. However, in a very large series of 3041 unselected CC, Wang et al. detected only $14(0.46 \%)$ cases with loss of SMARCB1 [19], which was significantly different from the first report $(p<0.001$, Xhi2 test). We use the same evaluation score as Wang et al. and in our main series of patients (cohort 1), which is limited to patients with stage III CC, the frequency of loss of SMARCB1 was not different from Wang et al. ( $p=0.2$, Xhi2 test), suggesting that the stage of CC has no or only limit correlation with this phenotype. Altogether in these three largest published series of $\mathrm{CC}$, the frequency of loss of SMARCB1 was $0.84 \%$ in 4880 patients, which likely reflects a real incidence below $1 \%$ in $\mathrm{CC}$ of any stage.

The frequency of loss of SMARCB1 seems to be higher in poorly differentiated CC. In fact, in the Italian series, 8 cases with loss out of 25 poorly differentiated CRC (32\%) were observed. Among the 511 high-grade cases of Wang, 12 were SMARCB1 negative $(2.35 \%)$. Briefly in Wang series, 12 of $14(85.7 \%)$ of the CRCs with SMARCB1 loss were high grade, compared to $19.6 \%$ of cases with preserved SMARCB1 staining $(p<0.001)$. In 3 of the 7 CRCs with focal SMARCB 1 loss, SMARCB1 stained in well-differentiated areas, and lost in areas of poor differentiation. In our large series of stage III CC, the only case with complete loss was a poorly differentiated CC: medullar type. Furthermore in our small independent series of poorly differentiated CC: $2 / 23$ (8.7\%) were SMARCB1 negative.

Although originally described in malignant rhabdoid pediatric tumors, SMARCB1 loss has now been reported in tumors from several localization, including the vulva [8], pancreas [2] and sinonasal tract [3]. In these sites, as well as in the gastrointestinal tract, SMARCB1 loss was reported to be associated with poor prognosis $[1,11,17,19]$. For instance, among the 134 cases of Pancione et al. the loss was associated with poorly differentiated tumors, most often metastatic, and with a lower survival, regardless of the MMR status. Wang et al. also observed that loss of SMARCB1 was associated with lower survival. Contrasting with these previous publications, we did not find any prognostic value of the loss of SMARCB1.

The previous studies retrospectively included patients with different UICC stages, and with heterogeneous treatment and follow up. By contrast, the 1695 that we analyzed were all at stage III, prospectively included in an international clinical trial. They all received FOLFOX adjuvant treatment and underwent the same follow up. For these reasons our results have a higher level of evidence, than the previously published series. However, the power of our series is limited by 
the low incidence of the SMARCB1 negative phenotype.

Only few cases of $\mathrm{CC}$ with rhabdoid histology and loss of SMARCB1 have been reported as yet. But they had common features: mean age 70 years old, localization of the proximal colon, metastases, poor prognosis, $B R A F$ mutated and dMMR status [1,12]. Wang's group showed that the loss of SMARCB1 in CC was associated with MSI-H status, and BRAF V600E mutation $(p<0.001)$. Molecular characteristics of the tumors of patients included in the PETACC8 series have already been published $[4,14,15]$. In these patients, loss of SMARCB1 was not associated with mismatch repair deficiency nor with BRAF V600E mutation. However, in our small series of poorly differenciated CC, one of the two cases with loss was rhabdoid, the other mixed (medullary and mucinous) and these 2 cases were $B R A F$ mutated and deficient for MMR. We can therefore speculate that SMARCB1 loss could be secondary to genetic instability. However no pathogenic somatic variant were detected within the coding sequences. The previously described hotspot mutation within intron 1 may also be responsible for the loss of SMARCB1 [16], but were also absent in the two cases. By contrast we detected a copy neutral $\mathrm{LOH}$ in one of the two cases. Further studies are required to determine the mechanism of SMARCB1 loss in poorly differentiated gastrointestinal carcinoma.

Genes of the SWI/SNF chromatin-remodeling complex are frequently altered in human cancers [20] and may be targeted by specific therapies in the future.

To date, there is no available targeted molecular therapy against pediatric rhabdoid neoplasms, but currently a recently developed EZH2 inhibitor, is undergoing clinical trial in children with rhabdoid tumor and loss of SMARCB1 (NCT02601937). EZH2 is a catalytic subunit of the histone methytransferase PCR2 which is blocked by the intact SWI/SNF complex [17]. When SMARCB1 is mutated, the accumulation of EZH2 promotes an undifferentiated state with maintenance of a "stem cell" program. Hedgehog-Gli pathway, Cyclin D1, Epidermal growth factor and Fibroblastic growth factor receptors [21] are other potential targets, which have been found to be up regulated in association with a disrupted SWI/SNF complex.

In view of the results obtained in the literature and in the independent cohort of undifferentiated CC studied in parallel, it seems advisable to evaluate the prognostic impact of the loss of expression of SMARCB1 by restricting the studied population to the poorly differentiated $\mathrm{CC}$. The IHC could allow a pre-screening of mutated patients, who may one day benefit from tar geted therapies.

In conclusion, loss of SMARCB1 expression is rare $(<1 \%)$ in stage III CC, but more frequent $(>5 \%)$ in poorly differentiated CC. Our study did not confirm the association of loss of SMARCB1 with MMR nor $B R A F$ status, neither with poorer prognostic.

\section{Acknowledgments}

The authors thank Dominique Péchaud, Yolaine Pothin, and Nathalie Terrones for technique contribution.

\section{Supplementary data}

The supplementary files are available to download from http://dx.doi.org/10.3233/CBM-190287.

\section{References}

[1] A. Agaimy, T.T. Rau, A. Hartmann and R. Stoehr, SMARCB1 (INI1)-negative rhabdoid carcinomas of the gastrointestinal tract: Clinicopathologic and molecular study of a highly aggressive variant with literature review, Am J Surg Pathol 38(7) (2014), 910-920.

[2] A. Agaimy, F. Haller, J. Frohnauer, I.M. Schaefer, P. Ströbel, A. Hartmann et al., Pancreatic undifferentiated rhabdoid car cinoma: KRAS alterations and SMARCB1 expression status define two subtypes, Mod Pathol 28(2) (2015), 248-260.

[3] A. Agaimy and W. Weichert, SMARCA4-deficient sinonasal carcinoma, Head Neck Pathol 11(4) (2017), 541-545.

[4] H. Blons, J.F. Emile, K. Le Malicot, C. Julié, A. Zaanan, J. Tabernero et al., Prognostic value of KRAS mutations in stage III colon cancer: Post hoc analysis of the PETACC 8 phase III trial dataset, Ann Oncol 25(12) (2014), 2378-2385.

[5] F. Bourdeaut, D. Lequin, L. Brugières, S. Reynaud, C. Dufour F. Doz et al., Frequent hSNF5/INI1 germline mutations in patients with rhabdoid tumor, Clin Cancer Res 17(1) (2011) 31-38.

[6] B. Brennan, C. Stiller and F. Bourdeaut, Extracranial rhabdoid tumours: What we have learned so far and future directions Lancet Oncol 14(8) (2013), 329-336.

[7] J. Calderaro, J. Masliah-Planchon, W. Richer, L. Maillot, P. Maille, L. Mansuy et al., Balanced translocations disrupting SMARCB1 are hallmark recurrent genetic alterations in renal medullary carcinomas, Eur Urol 69(6) (2016), 1055-1061.

[8] A.L. Folpe, J.K. Schoolmeester, W.G. McCluggage, L.M. Sullivan, K. Castagna, W.A. Ahrens et al., SMARCB1 deficient vulvar neoplasms: A clinicopathologic, immunohistochemical, and molecular genetic study of 14 cases, Am J Surg Pathol 39(6) (2015), 836-849.

[9] K. Kohashi, Y. Oda, H. Yamamoto, S. Tamiya, Y. Oshiro, T. Izumi et al., SMARCB1/INI1 protein expression in round cell soft tissue sarcomas associated with chromosomal transloca- 
tions involving EWS: A special reference to SMARCB1/INI negative variant extraskeletal myxoid chondrosarcoma, $A m J$ Surg Pathol 32(8) (2008), 1168-1174.

10] J. Masliah-Planchon, I. Bièche, J.M. Guinebretière, F. Bourdeaut and O. Delattre, SWI/SNF chromatin remodeling and human malignancies, Annu Rev Pathol 10 (2015), 145-171.

[11] M. Pancione, A. Remo, C. Zanella, L. Sabatino, A. Di Blasi, C. Laudanna et al.,The chromatin remodelling component SMARCB1/INI1 influences the metastatic behavior of colorectal cancer through a gene signature mapping to chromosome 22, J Transl Med 11 (2013), 297.

[12] M. Pancione, A. Di Blasi, L. Sabatino, A. Fucci, A.M. Dalena, N. Palombi et al., A novel case of rhabdoid colon carcinoma associated with a positive $\mathrm{CpG}$ island methylator phenotype and BRAF mutation, Hum Pathol 42(7) (2011), 1047-1052.

13] J. Taieb, J. Tabernero, E. Mini, F. Subtil, G. Folprecht, J.L. Van Laethem et al., Oxaliplatin, fluorouracil, and leucovorin with or without cetuximab in patients with resected stage III colon cancer (PETACC-8): An open-label, randomised phase 3 trial, Lancet Oncol 15(8) (2014), 862-873.

[14] J. Taieb, A. Zaanan, K. Le Malicot, C. Julié, H. Blons, L. Mineur et al., Prognostic effect of BRAF and KRAS mutations in patients with stage III colon cancer treated with leucovorin, fluorouracil, and oxaliplatin with or without cetuximab: A post hoc analysis of the PETACC-8 trial, JAMA On$\operatorname{col}(2016), 1-11$.

15] J. Taieb, K. Le Malicot, Q. Shi, F. Penault Lorca, O. Bouché, J. Tabernero et al., Prognostic value of BRAF and KRAS mutations in MSI and MSS stage III colon cancer, J Natl Cancer Inst 109(5) (2017).
[16] A. Tauziède-Espariat, J. Masliah-Planchon, L. Brugières, S Puget, C. Dufour, P. Schneider et al., Deep intronic hotspo variant explaining rhabdoid tumor predisposition syndrome in two patients with atypical teratoid and rhabdoid tumor, Eur J Hum Genet 25(10) (2017), 1170-1172.

[17] L. Tóth, Z. Nemes, S. Gomba, L. Asztalos, C. Molnár, C. An drás et al, Primary rhabdoid cancer of the ileum: A case report and review of the literature, Pathol Res Pract 206(2) (2010) $15 ; 110-115$.

[18] X. Wang, J.R. Haswell and C.W. Roberts, Molecular pathways: SWI/SNF (BAF) complexes are frequently mutated in cancer-mechanisms and potential therapeutic insights, Clin Cancer Res 20(1) (2014), 21-27.

[19] J. Wang, J. Andrici, L. Sioson, A. Clarkson, A. Sheen, M. Farzin et al., Loss of INI1 expression in colorectal carcinoma is associated with high tumor grade, poor survival, BRAFV600E mutation, and mismatch repair deficiency, Hum Pathol 55 (2016), 83-90.

[20] X. Wang, J.R. Haswell and C.W. Roberts, Molecular pathways: SWI/SNF (BAF) complexes are frequently mutated in cancer-mechanisms and potential therapeutic insights, Clin Cancer Res 20(1) (2014), 21-27.

[21] S. Wöhrle, A. Weiss, M. Ito, A. Kauffmann, M. Murakami, Z. Jagani et al., Fibroblast growth factor receptors as novel ther apeutic targets in SNF5-deleted malignant rhabdoid tumors PloS One 8(10) (2013), 77652. 